\title{
Comparative analysis of FACTS devices based on the comprehensive evaluation index system
}

\author{
Liu Jiankun ${ }^{1}$, Chen Jing ${ }^{1}$ and Qian Zhen ${ }^{2}$ \\ ${ }^{1}$ State Grid Jiangsu Electric Power Company Electric Power Research Institute, Nanjing 211103, Jiangsu Province, China \\ ${ }^{2}$ College of Energy and Electrical Engineering, Hohai University, Nanjing 211100, China
}

\begin{abstract}
Flexible AC transmission system (FACTS) has the control characteristics of efficiency, flexibility and reliability. The introduction of FACTS in power system is superior to any other control methods, and different FACTS devices have different features. In this paper, a comprehensive evaluation index system is developed to study a variety of comparisons on different FACTS devices. The proposed index takes power flow controllability as its main indicator, and loss reduction, static voltage stability improvement and load shedding reduction as complementary indicators. Finally, the peak load case in 2016 of a province is adopted for case studies. The results not only show that the proposed comprehensive evaluation index is systematic, scientific, practical, but also show the superiority of unified power flow controller (UPFC).
\end{abstract}

\section{Introduction}

At present, the primary equipments of grid framework are mainly based on the electromagnetic and mechanical technology. Its flexibility and controllability is limited, which makes it difficult to control the power flow, voltage and others quickly and flexibly. Therefore, the technology of FACTS provides a new technical means for power grid. The concept of FACTS was initially put forward by American scholar N. G. Higorani in 1986, which injected new vitality into the traditional transmission system as a transformative technology [1]. The core idea of FACTS is to enable the system electric parameters (such as voltage, phase angle and impedance) to change fast and flexibly under the premise of keeping the security, stability and reliability of power system, thus making the most of existing resources for distributing transmission power reasonably, reducing power loss and cost and improving the efficiency of the power grid operation [2].

According to the connection mode in power system, the FACTS devices can be divided into three types: a) shunt devices like static var compensator (SVC) and static synchronous compensator (STATCOM), which are mainly used for voltage control and reactive power flow control; b) series devices like thyristor controlled series capacitor (TCSC), thyristor controlled phase shifting transformer (TCPST) and static synchronous series compensator (SSSC), which are mainly used for the control of active power flow, the improvement of the transient stability and the inhibition of power oscillator; c) comprehensive devices like unified power flow controller (UPFC), which is a combination of a) and b). UPFC is by far the most powerful FACTS and has a broad application prospect. It can not only improve the power flow distribution effectively, but also improve the stability of power system. On December 11, 2015, the demonstration project of UPFC, which has the first independent intellectual property rights of China, is officially put into operation in Nanjing. The project provides a practical basis for the theoretical analysis of UPFC operation. What's more, the demonstration project of UPFC in Suzhou is also on agenda.

Based on the above discussion, this paper proposed a new comprehensive evaluation index, which takes power flow controllability as its main indicator, and loss reduction, static voltage stability improvement and load shedding reduction as complementary indicators. This paper is organized as follows. The selection of typical FACTS devices including TCSC, TCPST, STATCOM and UPFC, and the static mathematical modeling of FACTS devices are established in Section II; In Section III, the calculation of every single index is conducted and the comparative analysis for different FACTS devices is conducted through the calculation of the comprehensive evaluation index; Section IV presents simulation and results based on the example of the peak load case in 2016 of a province. Finally, conclusion is drawn in Section V.

\section{Matematical modelling of FACTS device}

\subsection{Static model of UPFC}

Generally, the back-to-back voltage source model is adopted to account for UPFC, as illustrated in Fig. 1. 
UPFC consists of a variable shunt voltage source $\dot{V}_{\mathrm{E}}$ along with the impedance $Z_{\mathrm{E}}$, and a variable series voltage source $\dot{V}_{\mathrm{B}}$ with the impedance $Z_{\mathrm{B}}$. It's assumed UPFC is installed at the s-side of line s-m. A dummy bus $r$ can be added at the end of UPFC, rendering the UPFC to be an independent branch for calculation. It has the ability to adjust the control variables - magnitude and phase angle of the series and shunt injected voltage sources simultaneously, or independently to regulate the voltage profile and power flow in the transmission line.

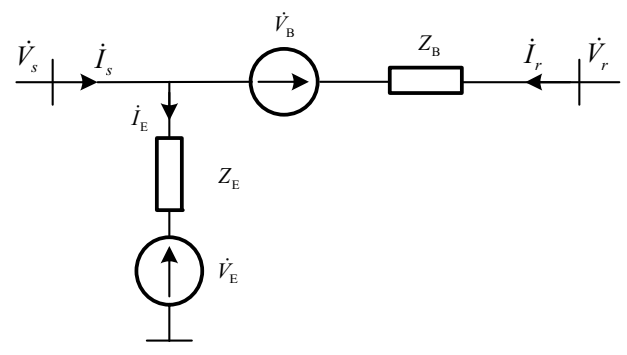

Figure 1. Dual Voltage Sources Model of UPFC.

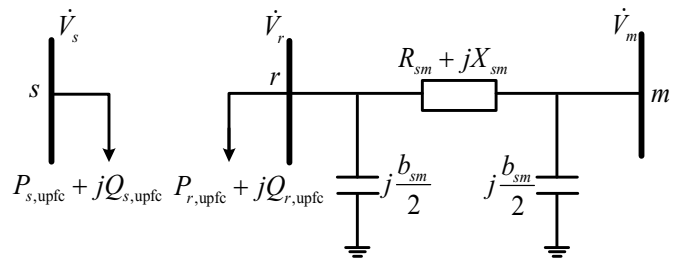

Figure 2. Diagram of UPFC branch equivalent.

When making calculations, the role of UPFC is equivalent to its injection power at each side of the branch, which are denoted as $P_{s, \text { upfc }}+j Q_{s \text {,upfc }}$ and $P_{r \text {,upfc }}+j Q_{r \text {,upfc }}$ in Fig.2. The equivalent injected power is written as:

$$
\left\{\begin{array}{l}
P_{s, \text { upfc }}+j Q_{s, \text { upfc }}=\dot{V}_{s} \dot{I}_{s}^{*}=\dot{V}_{s}\left(\dot{I}_{\mathrm{E}}-\dot{I}_{r}\right)^{*} \\
P_{r, \text { upfc }}+j Q_{r, \text { upfc }}=\dot{V}_{r} \dot{I}_{r}^{*}
\end{array}\right.
$$

\subsection{Static model of STATCOM}

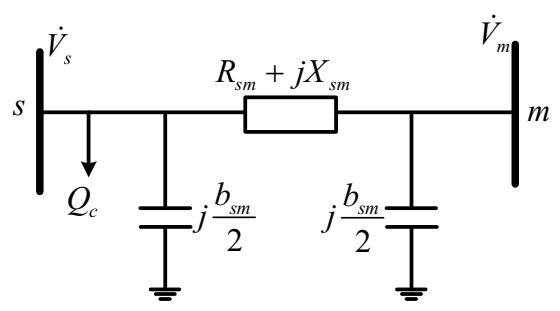

Figure 3. Diagram of STATCOM branch equivalent.

STATCOM is the type of shunt reactive compensation device, which targets at supporting the voltage magnitude profile. Hence, the STATCOM can be operated as a capacitive or inductive compensation by injecting or absorbing reactive power from the system to regulate voltage. It is regarded as a reactive power injection $Q_{\text {c }}$ in Fig.3.

\subsection{Static model of TCSC}

TCSC can be operated to control the line flow as an expected constant within a rational operation region by changing the equivalent line reactance fastly and continuously. Figure 4 shows the model of TCSC. The series variable reactance in Fig.4, $X_{\mathrm{c}}$, can be adjusted. The maximum value of $X_{\mathrm{c}}$ is decided by the capacity of TCSC. However, it is noted that active and reactive of the line are dependent. Therefore, active power flow is considered to be controllable.

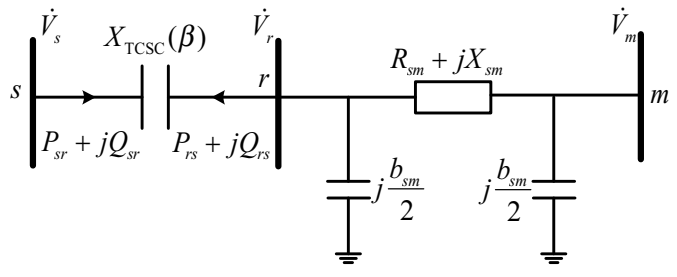

Figure 4. Diagram of TCSC branch equivalent.

\subsection{Static model of TCPST}

TCPST realizes rapid adjustment of the phase shifter through mechanical switch with thyristor. This fosters the wide application of TCPST. The equivalent circuit of TCPST is given in Fig. 5. It is equivalent as a series voltage source and a shunt current source. Power flow is controlled by adjusting the complex ratio. Similarly, active and reactive power cannot be controlled dependently, so active power line cab be regarded as controllable.

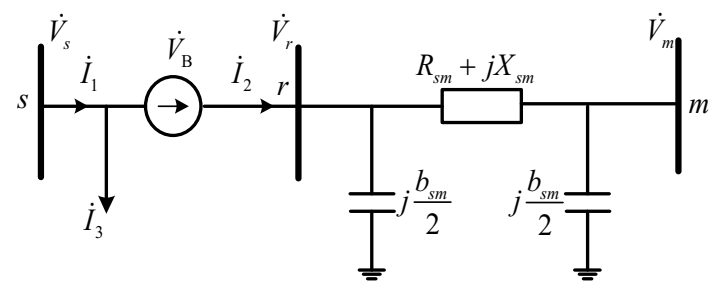

Figure 5. Diagram of TCPST branch equivalent.

\section{Comprehensive evaluation index}

In this part, a comprehensive evaluation index is proposed to compare the performance of the aforementioned FACTS devices. The proposed index is systematic, scientific and practical in assessing the vast series and shunt type devices. Moreover, the proposed index takes power flow controllability as its main indicator, and loss reduction, static voltage stability improvement and load shedding reduction as complementary indicators.

\subsection{Indicator of control region}


Three devices with the ability of series power flow control, namely TCSC, TCPST and UPFC, are compared. Figure 6 depicts the equivalent electric line installed with FACTS devices, the power flow of which is controlled by the series voltage source $\dot{U}_{p q}$. To simplify, it's assumed $\dot{U}_{i}=U_{i} \angle 0, \quad \dot{U}_{j}=U_{j} \angle(-\theta), \quad Z=R+j X$.

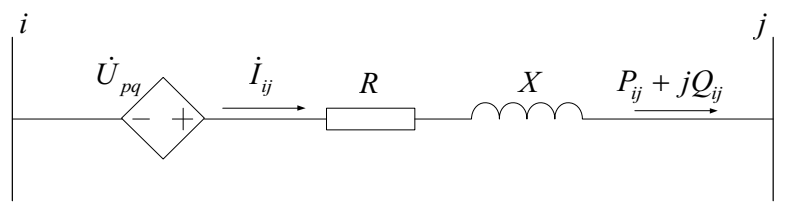

Figure 6. The equivalent electric line installed with FACTS devices.

Then the apparent line flow is written as:

$$
\dot{S}_{i j}=P_{i j}+j Q_{i j}=\dot{U}_{j}\left[\left(\dot{U}_{i}+\dot{U}_{p q}-\dot{U}_{j}\right) / Z\right]^{*}
$$

If $\dot{U}_{p q}=0$, the electric line is not controlled by FACTS. In this case, eq. (2) can be reformulated as:

$$
\dot{S}_{i j}=\dot{S}_{i j}^{0}=P_{i j}^{0}+j Q_{i j}^{0}=\dot{U}_{j}\left[\left(\dot{U}_{i}-\dot{U}_{j}\right) / Z\right]^{*}
$$

Equation (2) can also be written as:

$$
\dot{S}_{i j}=\dot{U}_{j}\left[\left(\dot{U}_{i}-\dot{U}_{j}\right) / Z\right]^{*}+\dot{U}_{j} U_{p q}^{*} / Z^{*}=\dot{S}_{i j}^{0}+\dot{S}_{i j}^{f a c t s}
$$

According to the formulations, the power flow controllable region is shown in Fig. 7, where the intersection point denotes original power flow point. The controllable region of TCSC is a section of arc going through the original point, while it is a segment (the slope is negative) going through the original point for TCPST. However, for UPFC, the controllable region is a filled circle, taking the original point as its center point.

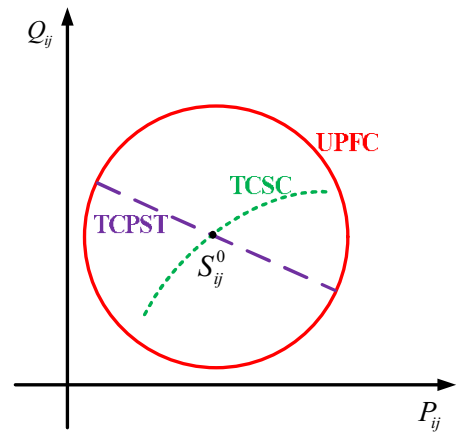

Figure 7. Diagram of power flow controllable region.

The area of the three controllable region is compared quantitatively, with the capacity of three each device being the same. It is assumed that the control area of TCSC is the area of the arc, and the rectangular area for TCPST. For UPFC, the control area is the whole circle.

\subsection{Indicator of power flow losses}

The reactive power optimization results are introduced to show their ability in reducing power flow losses.
Concretely, on the basis of conventional reactive power optimization model, some modifications are made in the admittance matrix and some equality constraints that are related to the buses, where FACTS devices are installed. In addition, the power balance equation of FACTS and capacity constraints are added into the model. Then, the modified optimization model is solved.

It is worth noting, no restrictions are set on control variables to enlarge the optimization space. In this way, the performance of FACTS devices in reducing power flow losses can be presented.

\subsection{Indicator of voltage stability}

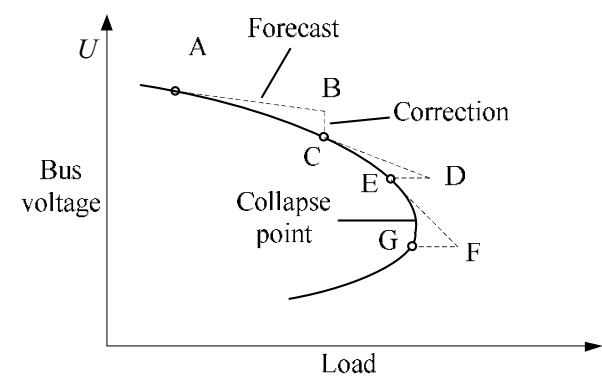

Figure 8. The calculation steps of the continuation power flow method.

The continuation power flow method is adopted in this paper to study the performance of FACTS in improving voltage stability. The calculation steps are illustrated in Fig. 8.

Loads are increased on the basis of conventional power flow as follows:

$$
\left\{\begin{array}{l}
P_{i s}-U_{i} \sum_{j \in N} U_{j}\left(G_{i j} \cos \theta_{i j}+B_{i j} \sin \theta_{i j}\right)-\lambda P_{d i}=0 \quad(i=1,2, \ldots, m) \\
Q_{i s}-U_{i} \sum_{j \in N} U_{j}\left(G_{i j} \sin \theta_{i j}-B_{i j} \cos \theta_{i j}\right)-\lambda Q_{d i}=0 \quad(i=1,2, \ldots, m)
\end{array}\right.
$$

Where $\lambda$ is load incremental coefficient; $P_{i s} 、 Q_{i s}$ are the predetermined active and reactive power injection at bus $i$; $P_{d i} 、 Q_{d i}$ is the incremental direction of active and reactive load; $\dot{U}_{i}$ is the voltage magnitude of bus $i ; \theta_{i j}$ is the voltage angle difference between bus $i$ and bus $j ; G_{i j}$ 、 $B_{i j}$ are the conductance and susceptance between bus $j$ and bus $j$, respectively; $n$ is the number of total buses; $\mathrm{m}$ is the number of PQ buses.

The continuation power flow method starts from the original working point and moves with the increase of load profile. The steps include prediction, correction and calculation of the complete curve. Finally, the maximum load incremental coefficient $\lambda_{\max }$ is obtained.

\subsection{Indicator of load shedding}

Load shedding reflects the insecure status of system operation and is suitable to be used as an indicator to assess the performance of FACTS devices. When the system security is endangered by an outage, a re-dispatch schedule can be obtained from optimal power flow (OPF) calculation to avoid the off-line limits. At the same time, load shedding is reduced as much as possible, if cannot be 
avoided. The optimal load shedding model is formulated as:

1) Objective function

$$
\min \sum_{i \in n_{d}} C_{i}
$$

2) Constraints (conventional constraints excluded)

$$
\begin{cases}P_{i s}-P_{D i}+C_{i}=0 & i=1,2, \ldots, n_{d} \\ Q_{i s}-Q_{D i}=0 & i=1,2, \ldots, n_{d} \\ 0 \leq C_{i} \leq P_{D i} & i=1,2, \ldots, n_{d}\end{cases}
$$

Where $P_{D i} 、 Q_{D i}$ are active and reactive load at bus $i ; C_{i}$ is the variable for load shedding; $n_{d}$ is the number of total buses.

\subsection{The comprehensive evaluation index}

A system of the comprehensive evaluation index is developed is this paper. The proposed index takes power flow controllability as its main indicator, and loss reduction, static voltage stability improvement and load shedding reduction as complementary indicators. The comprehensive evaluation index consists of four indicators, namely:

1)Controllable region, denoted as $C_{\text {region }}$, p.u.;

2)System power flow losses, denoted as $P_{\text {loss }}$, MW;

3)Maximum load incremental coefficient, denoted as $\lambda_{\max }$, dimensionless;

4)Load shedding, denoted as $L_{\text {loss }}$, MWh.

Denote the comprehensive evaluation index as $\rho$, it can be calculated as:

$$
\rho=\omega_{1} \mu\left(C_{\text {region }}\right)+\omega_{2} \mu\left(P_{\text {loss }}\right)+\omega_{3} \mu\left(\lambda_{\max }\right)+\omega_{4} \mu\left(L_{\text {loss }}\right)
$$

Where $\mu(\bullet)$ is syntropic normalization; $\omega_{1} 、 \omega_{2} 、 \omega_{3} 、 \omega_{4}$ are the weights of each indicator, and the sum of these is 1 .

\section{Numerical cases}

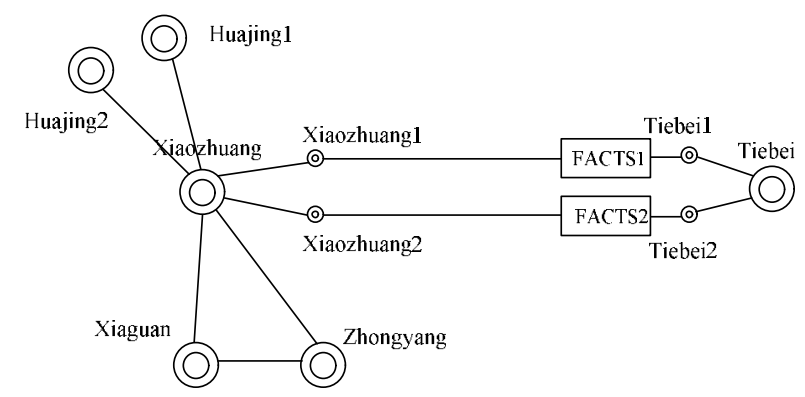

Figure 9. The system diagram installed with FACTS.

The peak load case in 2016 of a province is adopted for case studies. A comparison is made on the performance of the four FACTS devices. The system diagram installed with FACTS, including STATCOM, TCSC, TCPST and UPFC, is depicted in Fig. 9. The devices are installed at the north-side of Xiaozhuang-Tiebei double-line.

The obtained results are listed in Table 1.

Table 1. The comparative quantities of evaluation indexes of different FACTS devices.

\begin{tabular}{|c|c|c|c|c|c|}
\hline $\begin{array}{c}\text { Evaluation } \\
\text { index }\end{array}$ & $\begin{array}{c}\text { Original } \\
\text { situation }\end{array}$ & TCSC & TCPST & STATCOM & UPFC \\
\hline$C_{\text {region }} /$ p.u. & 0 & 0.365 & 1.106 & 0 & 10.401 \\
\hline$P_{\text {loss }} / \mathrm{MW}$ & 1056.5 & 1050.9 & 1049.8 & 1037.4 & 1021.6 \\
\hline$\lambda_{\max }$ & 1.82 & 1.84 & 1.86 & 1.90 & 1.92 \\
\hline$L_{\text {loss }} / \mathrm{MWh}$ & 9340 & 9024 & 8790 & 8246 & 7634 \\
\hline
\end{tabular}

In Table $1, C_{\text {region }} 、 \lambda_{\max }$ are positive relevant to the evaluation index while $P_{\text {loss }} 、 L_{\text {loss }}$ are negative relevant. It is noted that the weighting factor can be set according to real situations, and is set to 0.25 in this paper. The evaluation results after syntropic normalization is shown in Table 2.

Table 2. The calculation results of the comprehensive evaluation index of different FACTS devices.

\begin{tabular}{|c|c|c|c|c|c|}
\hline $\begin{array}{c}\text { Comprehen- } \\
\text { sive evalua- } \\
\text { tion index }\end{array}$ & $\begin{array}{c}\text { Origi- } \\
\text { nal } \\
\text { situa- } \\
\text { tion }\end{array}$ & $\begin{array}{c}\text { TCS } \\
\mathbf{C}\end{array}$ & $\begin{array}{c}\text { TCPS } \\
\text { T }\end{array}$ & $\begin{array}{c}\text { STAT- } \\
\text { COM }\end{array}$ & $\begin{array}{c}\text { UPF } \\
\text { C }\end{array}$ \\
\hline$\rho$ & 0.683 & 0.703 & 0.729 & 0.725 & 1 \\
\hline
\end{tabular}

According to Table 2, we have following conclusions: 1) The results of FACTS's comprehensive evaluation index are bigger than the original situation, which suggests that FACTS devices can effectively improve the performance, the power supply capacity, economy, safety and reliability of system.

2) UPFC has strong ability of control, which not only can control the active power, reactive power of line flow and the voltage of shunt bus independently, but also can control the above parameters jointly. Judging from the results of the comprehensive evaluation index, UPFC is significantly better than the other FACTS devices.

\section{Conclution}

In this paper, a system of the comprehensive evaluation index is developed to study a variety of comparisons on different FACTS devices. The index takes power flow controllability as its main indicator, and loss reduction, static voltage stability improvement and load shedding reduction as complementary indicators. By selecting different types of FACTS devices, the calculation of the comprehensive evaluation index has been carried out, and the following conclusions could be obtained:

1) Regardless of single or comprehensive evaluation index, UPFC is significantly better than the other FACTS devices, which has important research significances to the UPFC project both in Jiangsu and Suzhou.

2) The proposed comprehensive evaluation index is a combination of the technical indicator (control region), the economic indicator (loss reduction), the safety 
indicator (static voltage stability improvement) and the reliability indicator (load shedding reduction), which is a systematic, scientific and practical evaluation index.

\section{References}

1. N. Hingorani. Future opportunities for electric power systems[J]. Power Engineering Review. IEEE, 1987, 7(10): 4-5.

2. N. G. Hingorani and L. Gyugyi, Eds. Understanding FACTS Concepts and Technology of Flexible AC Transmission Systems[M]. Wiley-IEEE Press, 2000.

3. Zhang Xiaoping. Modeling of FACTS in power flow and optimal power flow analysis[J]. Sutomation of Electric Power Systems. 2005, 29(16): 22-29.

4. C. A. Canlzares, Z. T. Faur. Analysis SVC and TCSC Controllers in Voltage Collapse[J]. IEEE Trans. Power Systems. Vol. 14, No. I, February, 1999: 158-165.

5. Nasri A, Conejo A J, Kazempour S J. Minimizing wind power spillage using an OPF with FACTS
devices[J]. IEEE Transactions on Power Systems. 2014, 29(05): 2150-2159.

6. M. A. Furini and P. B. De Araujo. A comparative study of the damping oscillation function of tcsc and upfc $[\mathrm{C}]$. Transmission and Distribution Conference and Exposition: Latin America, 2008 IEEE/PES. 2008: 1-6.

7. Enrigue Acha, Claudio R. Fuerte-Esquivel, Hugo Ambriz-Perez, et al. FACTS Modeling and Simulation in Power Networks[M]. John Wiley \& Sons Ltd, 2004: 200-216.

8. R. Nelson, J. Bian, S. L. Williams. Transmission series power flow control[J]. IEEETransactions on Power Delivery. 1995: 437-443.

9. Mittelstaedt M, Barrios H, Schnettler A. Identification of critical states regarding voltage stability by using a Continuation Power Flow combined contingency analysis[C]// PowerTech, 2015 IEEE Eindhoven. IEEE, 2015: 256-261.

10. Pavel Etingov, Alexandre Oudalov, Nikolai Voropai. Coordinated emergency control of load shedding and FACTS devices[C]. // PowerTech, 2005 IEEE Russia. 2005: 1-8. 